Review

\title{
Paradigms in Integrative Medicine and the Place of Clinical Hypnosis
}

Donald Moss ${ }^{*}$

College of Integrative Medicine and Health Sciences, Pasadena, CA, USA; E-Mail: dmoss@saybrook.edu

* Correspondence: Donald Moss; E-Mail: dmoss@saybrook.edu

Academic Editor: Giuseppe De Benedittis

Special Issue: Hypnosis: from Neural Mechanisms to Clinical Practice

OBM Integrative and Complementary Medicine 2020, volume 5 , issue 1

doi:10.21926/obm.icm.2001007
Received: January 20, 2020

Accepted: February 11, 2020

Published: February 19, 2020

\begin{abstract}
The face of illness has changed with progress in public health, immunizations, and antibiotic medication. Today, medical clinics are more likely to see patients with chronic illnesses, stress-related conditions, and complex bio-psycho-social conditions. This is a global trend, affecting patients worldwide, as Western lifestyle and diet have produced increases in chronic lifestyle-based disorders. Integrative medicine, which attends more fully to psychosocial dimensions of illness, appears to offer promise for today's patient. One might expect hypnosis to assume a central place in integrative healthcare settings, because hypnosis is evidence-based, person-centered, and attuned to subjective and life-historical dimensions of the human being. However, hypnosis currently receives relatively little attention in integrative medicine circles, and hypnosis is underutilized in spite of the availability of many effective clinical protocols for a wide range of medical disorders. This article introduces emerging paradigms in integrative healthcare and suggests that the field of hypnosis must increase attention to these paradigms and improve educational preparation for hypnosis practitioners to function within these paradigms.
\end{abstract}

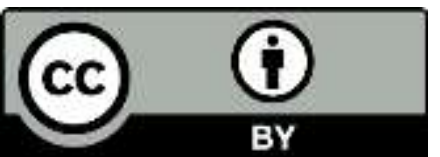

(C) 2020 by the author. This is an open access article distributed under the conditions of the Creative Commons by Attribution License, which permits unrestricted use, distribution, and reproduction in any medium or format, provided the original work is correctly cited. 


\section{Keywords}

Clinical hypnosis; integrative medicine; paradigms; lifestyle medicine; interprofessionalism; competencies

\section{Background}

\subsection{Changing Face of IIIness}

The face of illness has changed [1, 2]. In 1900 physicians faced the scourge of acute conditions, which often killed quickly. Infectious diseases, bacterial parasites, and unhealed physical trauma all took a toll on human beings with heavy mortality rates for untreatable conditions. The impact of public health, immunization, and antibiotic medications have reduced the illnesses of the past to the margin in health care. Today's primary care clinic rarely sees typhoid, cholera, smallpox, or polio.

Instead, patients approach the clinic today with chronic illnesses, stress-related conditions, and in general complex bio-psycho-social conditions. Authorities in medicine have been calling for several decades for a more integrative approach to health, to address the contributions of the biological, psychological, and social dimensions to illness [3-5]. Hypertension, depression, anxiety, diabetes, fibromyalgia, chronic fatigue, and chronic pain are more common problems in the $21^{\text {st }}$ century, each reflecting complex etiology, with life stress and lifestyle influencing both onset and progression [2].

These problems are global, spreading rapidly into the developing world, with the influences of urbanization, Western diet and lifestyle, and an aging world population. The World Health Organization has highlighted the trend for non-communicable diseases, largely chronic conditions, to burden populations in developing nations, which now are forced to budget for healthcare systems to deal with the old problems of infectious diseases and bacterial parasites while simultaneously treating epidemic numbers of patients with lifestyle-related problems [6-8].

Several authors have articulated a vision of integrative healthcare. Harold Koenig, a leader in the importance of religion and spirituality in health care, commented that:

Patients want to be seen and treated as a whole person, not as diseases. A whole person is someone whose being has physical, emotional, and spiritual dimensions. Ignoring any of these aspects of humanity leaves the person incomplete and may even interfere with healing [9].

Andrew Weill, who founded the first fellowship for physicians in integrative medicine, defines integrative medicine as:

...healing-oriented medicine that takes account of the whole person, including all aspects of lifestyle. It emphasizes the therapeutic relationship between practitioner and patient, is informed by evidence, and makes use of all appropriate therapies [10].

Since 1946, the WHO has called for treating the whole person, defining health as "a state of complete physical, mental and social well-being and not merely the absence of disease or infirmity" [11]. 
Integrative medicine has been promoted as a response to the global healthcare crisis, because it is focused broadly on psychosocial dimensions of illness, is person-centered, and emphasizes dietary, behavioral, and lifestyle change.

\subsection{The Place of Hypnosis in Integrative Healthcare}

One might expect a central role for hypnosis in integrative healthcare. Hypnosis draws on the inner resources of the person. It is delivered within the context of the patient-provider relationship. It has an extensive research base [12-15]. It addresses dimensions of the personwith-illness beyond the bio-medical, including life-historical factors such as adverse childhood experiences [16].

Hypnosis has been shown to facilitate habit change, including health-risk habits, with smoking cessation and smoking relapse prevention [17-20]. Hypnosis has been shown to prepare patients effectively for surgical procedures and other medical interventions, to a degree producing costsavings in overall care $[21,22]$. Hypnosis also moderates the adverse effects of medical procedures such as radiation therapy for cancer patients [23]. Hypnosis facilitates pain management for a wide variety of pain types, including acute pain, chronic pain, and pain accompanying medical procedures $[15,24,25]$. Hypnosis and hypnotic language can also be utilized by hospital and clinic personnel, with significant improvements in patient satisfaction [26, 27].

Early in the emergence of integrative medicine, [28] reviewed the relevance and credibility of hypnosis for inclusion in integrative medicine. He reported that NIH regards hypnosis as a complementary and alternative intervention. He reviewed outcome data on 17 medical applications of hypnosis, from allergy to urology, and concluded that "patients treated with hypnosis experienced substantial benefits for many different medical conditions" (2005, p. 511).

Nevertheless, the current dialogue on integrative medicine gives only sporadic attention to hypnosis and hypnosis remains underutilized in healthcare. The National Center for Complementary and Integrative Healthcare, the successor to the National Center for Complementary and Alternative Medicine, does not mention hypnosis in its discussion of mindbody practices, while chiropractic, acupuncture, yoga, and guided imagery are all mentioned [29]. Hypnosis is not listed among the 10 most commonly used complementary health approaches in national health statistics reporting [30], The American College of Physicians updated its recommendations for acute, sub-acute, and chronic low back pain in 2017, and called for noninvasive alternative treatments to be attempted before surgery, and further recommended a variety of alternative therapies to be implemented before medication. The guidelines differed for acute low back pain, chronic low back pain, and radicular low back pain, and included acupuncture, massage, heat, yoga, EMG biofeedback, and mindfulness, but not hypnosis [31]. Similarly, Lake, a leader in the integrative mental health (IMH) movement, gives minimal attention to hypnosis as a modality for $\mathrm{IMH}$, while emphasizing acupuncture, herbal and nutritional medicine, yoga, and meditation [32-34].

\section{Emerging Paradigms of Integrative Medicine}

In the remainder of this paper I will provide an overview of several emerging paradigms in the field of integrative healthcare. I propose that for hypnosis to assume a significant role in 
integrative healthcare, we must increase our attention to these paradigms, and improve educational preparation for hypnosis practitioners to function within these paradigms. Readers may also wish to consult [35] for a qualitative analysis of several definitions of integrative medicine and health.

\subsection{Integration within the Traditional Medical Hierarchy}

Under this paradigm, the physician remains the primary provider and will typically prescribe a biomedical regimen of medication, to be supplemented with complementary and behavioral interventions. Psychologists, nutritionists, exercise physiologists, acupuncturists, Reiki therapists, and other complementary practitioners will be included within the medical home or referral networks will be established between the medical home and the complementary providers. Those complementary therapy practitioners co-located within the medical home will often function as physician extenders, implementing physician-initiated treatment orders.

This paradigm continues the traditional assumption that the primary intervention for a patient complaint is bio-medical, and the medical physician is the best arbiter of choice of treatment. The positive advantage of the hierarchical model is that the supervising physicians may better integrate an understanding of the evidence-base of available therapies, when developing the treatment plan for the patients' disorders.

The disadvantage of this paradigm is that for many chronic conditions and functional disorders, a complementary or behavioral intervention delivered early in onset of disorder may reverse or at least manage the disorder and hinder the progression to chronicity. Too often medical providers delay referral until the pharmaco-therapies and medical procedures have been implemented for months or years, by which time the patient's condition often becomes more chronic and resistant to treatment of any kind. Biofeedback, hypnosis, or the Feldenkrais method, alone or in conjunction with lifestyle modifications, may often provide an effective intervention for emerging headache conditions, while the same therapies are challenged to address a "chronic daily headache," of ten years' duration, often exacerbated by a rebound patter of worsening head pain as blood levels of narcotic analgesics fade. Further, no one, including the physician, can reliably predict which complementary intervention might dramatically relieve the disorder.

\subsection{Interprofessionalism: A New Communications Paradigm}

Many advocates of Integrated Care believe that it can be optimized with interprofessional teams working in a single setting [36]. Co-location within a single setting enhances communication and increases patient follow through on referrals to other disciplines. Diverse professionals work on a collaborative basis in a single pediatric, family practice, or specialty medicine setting. Decision making is carried out through communication across disciplines and engaging the patient integrally in the process.

The World Health Organization advocates for interprofessionalism in health profession education and in clinical practice:

The World Health Organization (WHO) and its partners recognize interprofessional collaboration in education and practice as an innovative strategy that will play an important role in mitigating the global health workforce crisis [37]. 
There are challenges for the interprofessional model. This model challenges all healthcare providers to better communicate with one another and the patient. At a time when corporate medicine drives a reduction in minutes spent per patient encounter, interprofessionalism demands time for communication among professionals and with the patient.

In addition, the evidence-base for many complementary and allied health interventions is weak and not sufficiently persuasive for medical personnel. This undermines the confidence of medically oriented professionals in giving full credibility to complementary modalities. The funding for large-scale multi-center trials for new medications comes from the profits of pharmaceutical sales, whereas in the complementary therapy domain, comparable funding sources are not available. Although publications of research, including some well-funded larger studies, have increased in recent years, overall the studies on hypnosis, acupuncture, and other complementary therapies tend to utilize much smaller samples and blinding is less feasible, when the patient's awareness is part of the therapies' pathways for action. Fortunately, the occasional dramatic improvement of medical patients with integrative care including complementary therapies can be persuasive for physicians, nurses, and other biomedically oriented members of the treatment team $[1,2]$.

\subsection{The Smörgåsbord Paradigm}

In effect, many integrative medicine programs operate on a "Smörgåsbord" principle. Patients are offered a variety of mainstream and complementary treatments, and patients select those that suit their preferences. Since much of the CAM delivery system is market driven and outside mainstream healthcare, patient preference is often the primary factor driving treatment selection.

Patient preferences are a key element in all evidence-based care, but it is critical that current knowledge on the efficacy of the various options is communicated to the patient making the choices. Research suggests that finding reliable information on complementary and alternative treatments is challenging, patients are confused by conflicting research reports and claims, and patients may be most strongly influenced by testimonials from other patients [38]. Patients often would prefer to receive information about CAM from trusted sources, such as their physicians, yet physicians are often not equipped to provide such information [39].

As a consequence, patient choices of complementary therapies may not be in synch with the evidence base on the therapies. Moves to integrate complementary therapies into mainstream medical institutions may moderate these effects of disinformation and lack of information, yet patient preference will remain an element in informed medical decision making.

\subsection{Epigenetics as a Paradigm for Integrative Medicine}

Bruce McEwen [16], a leader in contemporary stress theory, has promoted epigenetics as a paradigm for integrative medicine. Epigenetics studies how multiple variables influence the translational process determining which of the potential traits carried in an organism's genetic code actually get expressed. Everything occurring during the organism's life cycle can modify gene expression, to the benefit or the detriment of health. Stress, nutrition, drugs, and negative emotion can all adversely affect gene expression and health. Animal research indicates that epigenetic effects may carry over transgenerationally from the original organism, "either through 
the germline or through reoccurring environmental conditions," suggesting a long-term effect for early experiences [40].

According to McEwen's model, early life experiences shape the trajectory of health and disease, influencing epigenetics. Early intervention is critical to minimize the damage caused by adverse childhood experiences (ACEs) - including abuse, neglect, and poverty -- with their lifelong health consequences. This paradigm demands interventions long before the patient presents with symptoms. McEwen emphasizes that the "lived experiences" of the person are important, since the same objective event may take on divergent significance or meaning for those impacted by it. Personal experiences, and the meaning the individual endows these experiences with, all enhance or inhibit the response to healthcare. Stress, allostatic load, and overload all can interact with genetic disposition toward bio-medical disorders.

In McEwen's model, physical exercise, social integration, and finding meaning in life can all optimize epigenesis and enhance the positive plasticity of the nervous system. Behavioral interventions, cognitive behavioral therapies, and mindfulness training can moderate the symptoms of chronic illness, enhance neurogenesis, and improve the regulation of mood and emotion, reducing negative epigenetic forces and enhancing positive epigenesis [16].

\subsection{The Lifestyle Medicine Paradigm and the Health Coaching Paradigm}

I have grouped these overlapping paradigms together, because they complement one another so well. Lifestyle medicine has emerged as a new movement in healthcare, based on decades of research showing that lifestyle factors shape both the onset and progression of chronic illnesses and conditions. Health coaching is a discipline that has emerged, to assist human beings to make lifestyle choices, reducing health risk behaviors and enhancing salutogenic behaviors.

\subsubsection{Lifestyle Medicine}

Sagner, et al. [41], published a definition of lifestyle medicine endorsed by the American College of Lifestyle Medicine, the Australian Lifestyle Medicine Association, and the European Society of Lifestyle Medicine:

Lifestyle medicine is a branch of evidence-based medicine in which comprehensive lifestyle changes (including nutrition, physical activity, stress management, social support and environmental exposures) are used to prevent, treat and reverse the progression of chronic diseases by addressing their underlying causes [41].

Dean Ornish conducted many of the early studies that led to the integrative medicine movement. Ornish developed a multi-modal protocol including whole foods, plant-based (vegetarian) diet, smoking cessation, physical exercise, stress management, yoga, and meditation. Patients who completed this program showed measurable and clinically significant improvements in their cardiovascular condition. Patients with cardio-vascular illness attained reductions in triglycerides, cholesterol, blood pressure, and increases in myocardial perfusion [42-44]. Many patients in Ornish's lifestyle medicine studies showed measurable opening of coronary artery blockages, through reversal of plaque formation. Following the Ornish program, cardiac patients with depressive illness also showed significant improvements in depressive mood [45]. 
Sagner and colleagues described the essence of lifestyle medicine. Evidence has mounted for decades that nine lifestyle-related factors and lifestyle-induced dysfunctions account for $90 \%$ of cardiovascular illness: "smoking, excessive alcohol consumption, physical inactivity, poor diet, stress, hypertension, and poor blood lipids" [41]. The message is clear: Better health lies in the direction of correcting these nine factors. The frustration is that decades have passed, and the same correctable lifestyle variables continue to cause needless cardiovascular deaths [46].

Healthcare professionals know the behavioral changes needed to enhance health and prevent illness; patients do not make the changes. The huge published research on "medical nonadherence" illustrates the magnitude of the problem. Patients frequently do not follow prescribed regimens of medication, as well as other physician recommendations. In chronic illness, 30-50 \% of medications are not taken as prescribed and medication nonadherence is associated with 125,000 deaths annually [47]. Non-adherence when physicians recommend behavioral and lifestyle change falls in similar ranges. According to one recent study, only from 35 to $63 \%$ of patients make positive behavioral changes following physician recommendations, although those reporting more trust in the physician make more changes [48].

David Katz sums up the message of lifestyle medicine:

We let this happen, every day. We aid it, and we abet it - every day. And all the while, we devise new drugs and devices, new stents and statins - and we learn CPR - to contend with diabetes that doesn't need to happen fully 90 percent of the time or more, heart disease that doesn't need to happen fully 80 percent of the time or more. We seem to accept that it's a midlife right of passage: angioplasty, or CABG? Take a number - have a nice procedure - you can wait in the fast food restaurant - next! [46]

\subsubsection{Health and Wellness Coaching}

Health and wellness coaching is the emerging discipline that applies research-based knowledge and techniques to assist human beings to modify lifestyle, reduce health risk behaviors, and increase health-enhancing behaviors $[49,50]$. Health and wellness coaching optimizes the human capacity to make change. Coaching is a natural partner with lifestyle medicine, since health coaches facilitate patients to make exactly the kinds of lifestyle changes demanded by lifestyle medicine.

The coach begins by inviting the patient into a collaborative "alliance for health." The coach uses motivational interviewing to mobilize the patient's own motivations and goals, assists patients to establish their own goals. Many authors within the coaching field emphasize selfdetermination theory [51].

Self-determination theory holds that the more autonomous a behavior is, the more personal commitment is mobilized [52]. Accordingly health and wellness coaches assess the patient's current readiness for change, and proceed in a step-wise fashion, beginning with small selfdirected changes, and moving to more demanding changes only when the individual has experienced some initial successes with small steps [53]. 


\subsection{The Functional Medicine Paradigm}

Functional medicine is a widely used paradigm within the larger field of integrative medicine. Functional medicine brings together the healing powers of nutrition with laboratory assessment, to identify specific deficits in the body that can be addressed nutritionally. It is clear that nutritional advice does not produce the same effects in all patients, even to those with the same diagnosis. This is the principle of biochemical individuality. Interventions must take into account this individual's history, biochemistry, genetic makeup, and nutritional deficiencies and sensitivities [54]. The goal is what is variously called "personalized medicine" or "precision medicine;" the treatment plan is unique for precisely this biochemically unique human being.

Functional medicine practitioners utilize a variety of blood and stool tests to identify any mineral or nutritional deficiencies along with food allergies and insensitivities. The practitioner assesses the current state of the microbiome of this individual, the genetic material of all of the microbes that reside in the human body [55]. This allows nutritional recommendations to be personalized for the patient.

Functional medicine includes attention to both nutrigenomics and nutrigenetics. Nutrigenomics investigates how nutrients can modify the regulation of gene expression. Diet-regulated genes play a role in the onset and progression of specific diseases. Emerging research has shown that nutrigenomics has potential for preventing, mitigating, or treating chronic disease, and certain cancers, through small but strategic dietary changes.

Nutrigenetics investigates how genetic variation influences the interaction between nutrition and disease. Research on nutrigenetics offers further tools to create a more individualized/personalized medicine based on understanding an individual's nutritional needs, health status, and genotype. In a large-scale study, those participants who had a genetic variant rs1885988 in the MT1F3 region showed greater weight loss from intensive lifestyle interventions than did those without this genetic variant [56]. The genetic evidence becomes a tool for supporting the choice of this intervention.

In this area of nutrigenetics and nutrigenomics, the causal pathways are reciprocal: Genetic markers predict response to interventions, but interventions also blunt the effects of genetic markers. In the so-called "Pounds Lost investigation," individuals carrying the A Obesity risk allele at FTO rs1558902 showed greater weight loss on a high protein diet [57]. In another study, those research participants who had a genetic marker at rs79903146 at TCF7L2, showed more frequent progression to diabetes. However, those participants with this marker who also received the intensive lifestyle intervention, showed lower frequency of developing diabetes [58].

There is also extensive research evidence for reciprocal interactions among life stress, the physiological stress response, digestive function, and the gut. Stress can cause or exacerbate digestive system symptoms, including heartburn, abdominal cramps, or loose stools. Keefer and Blanchard [59] estimated that $60 \%$ of patients with IBS have Generalized Anxiety Disorder, and $20 \%$ have depression. The microbiome may cause or exacerbate anxiety and traumatic experiencing; conversely, use of a probiotic regulating the microbiome can reduce anxiety and depression [60].

Stress influences food choices: Stress and depression are associated with less fresh fruit consumption as well as greater snack food intake, and the seeking of hyperpalatable foods [61]. Stress also induces metabolic changes that promote weight gain and body fat mass [61]. 
Inflammation-enhancing diets can increase depressive symptoms and pro-inflammatory responses to stressors. Stress enhances maladaptive metabolic responses to unhealthy meals. For example, total triglycerol (TG) and low-density-lipoprotein-TG areas were $50 \%$ or more higher during stress than under control conditions [62]. Accordingly, a comprehensive functional medicine plan should include mind-body elements, such as relaxation training or hypnosis, to counter the effects of stress on digestion, food choice, and metabolism.

In summary, functional medicine has drawn on the full powers of current science and laboratory testing to personalize the treatment plan for the individual patient. Practitioners guide patients to eliminate some foods, increase others, and in many cases the practitioners also prescribe nutritional or mineral supplements to address conditions identified in the laboratory. The research in this domain remains weak and inconsistent, yet most functional medicine clinicians report that many of their patients have shown dramatic improvements, enough to inspire further research on functional medicine.

\subsection{The Competencies Paradigm}

Education in the health sciences today is moving toward a competency-based approach, which parallels evidence-based approached to practice. In integrative medicine, we showed above that the interprofessionalism paradigm emphasizes practitioners learning better communication skills, developing an understanding and acceptance for both the patient's perspective and the viewpoints of both mainstream and complementary healthcare disciplines. Accordingly, competencies for integrative medicine training will include communication skills and sensitivity to divergent professional cultures. Research on core competencies for integrated and integrative care has focused on integration of medical and "behavioral health" services. For example, Miller and colleagues concluded that:

Competence as a licensed behavioral health provider working in primary care refers to the knowledge, skills, and attitudes-and their interconnectednessthat allow an individual to perform the tasks and roles in that setting [63].

At least two organizations have identified competencies that support integration of professions in one setting.

The Colorado Consensus Conference took place in November 2015, and identified eight competencies to support highly integrated practices, where behavioral health professionals are onsite and integrated into the care team. The following are the headings for the eight competencies; further detail on each competency is available in the conference report [60]:

- Identify and assess behavioral health needs as part of a primary care team.

- Engage and activate patients in their care.

- Work as a primary care team member to create and implement care plans that address behavioral health factors.

- Help observe and improve care team function and relationships.

- Communicate effectively with other providers, staff, and patients.

- Provide efficient and effective care delivery that meets the needs of the population of the primary care setting.

- Provide culturally responsive, whole-person and family-oriented care. 
- Understand, value, and adapt to the diverse professional cultures of an integrated care team.

Another organization produced a similar set of competencies. The Annapolis Coalition on the Behavioral Health Workforce is a non-profit organization focused on strengthening the recruitment, retention, training, and performance of the behavioral health workforce. This group, in collaboration with SAMSHA, published a report on "core competencies for integrated primary and behavioral healthcare" [64].

The development of core competencies provides a paradigm and a training framework for developing a new healthcare workforce, comprised of professionals who are better prepared to interact across disciplinary boundaries, engage the patient in health care decisions, listen empathically to colleagues and patients, and produce true and more seamless integration of mainstream and complementary health modalities in one setting.

\subsection{The Eco-Systems Paradigm for Health and Healthcare}

Sagner et al. [41] suggested that our environment can be morbidogenic or salutogenic. The WHO is promoting attention to scientifically measurable variables in the environment, the "physical, chemical and biological determinants of health external to a person" [65]. The human being is always embedded in an eco-system or environment, and that environment can contribute to illness or health. This is reminiscent of the words of Ralph Waldo Emerson, who wrote in his essay Nature that "In the woods, I feel that nothing can befall me ... which nature cannot repair" [66].

There are a number of eco-systems models for health at this time, but I will take the example of "forest medicine" and "forest bathing." One of the stimuli for global lifestyle-based illness is rapid urbanization and estrangement from nature. Forest medicine emerged in Japan to address this estrangement from nature and has become a global movement. Forest medicine and other forms of nature therapy prescribe immersion - "forest bathing" - in natural environments as a form of therapy. Organized programs provide transport for urban dwellers to protected forest environments and these individuals are immersed in the forest for periods of several days.

In Japan, the Shinrin-Yoku movement began in the 1980's and has gained a widespread following. Shinrin-yoku literally translates as taking in the forest atmosphere or "bathing" in the forest. Researchers in both Japan and South Korea have promoted forest exposure for preventative health and wellness and also as a treatment for chronic illness. One of the leading researchers in this area, Yoshifumi Miyazaki, summarized much of the Japanese research in this area in a 2014 article. He reported that time spent in forest environments in 35 different forests in Japan was shown to produce a $12.4 \%$ decrease in cortisol levels, a $7.0 \%$ decrease in sympathetic nervous activation, and a $5.8 \%$ decrease in heart rate. He claimed a $55 \%$ increase in parasympathetic activation, indicating substantial physiological relaxation, and a dramatic increase in immune function that persisted for one month after the forest exposure [67]. A study by Li [68] measured natural killer cell activity in 12 research participants, who were exposed to a forest environment for a weekend. The study reported significant increases in natural killer cell activity in the study participants, lasting a month. Another study, reported by Bing Bing et al. [69], utilized a randomized control design and immersed the experimental group in a forest environment while the control subjects remained in the city. The forest immersion group showed 
decreased levels of pro-inflammatory cytokines and stress hormones and decreases in negative mood as measured on the POMS.

The forest medicine movement has gained support in Europe, and research is beginning there as well. There is now a Europe-based International Congress on the Forest and its Potential for Health, scheduled annually, and promoting both research and clinical applications of forest medicine (https://www.selvans.ong/congress2019/?lang=en). Much of the research on the health effects of forest immersion consists of small studies, some of less rigorous design [70]. Nevertheless, with the shared consensus that stressful urban environments are a factor undermining health, initiatives to integrate environmental factors into health are welcome.

\section{The Significance of the Integrative Medicine Paradigms for the Hypnosis Profession}

Hypnosis deserves a place in integrative healthcare. As summarized above, it is evidence-based, person-centered, effective in modifying habits and lifestyle, and includes a variety of protocols with well-documented efficacy for medical disorders, and effective applications for pain management, surgery preparation, obstetrics, oncology, and many other areas.

Reviewing the paradigms for integrative medicine included above, I propose the following guidelines for professional education in hypnosis, and for health professionals wishing to increase their role in integrative care:

1. Medical hierarchy paradigm. Because the hierarchical paradigm prevails in many settings, it is important for hypnosis practitioners to become more informed about the hierarchy, the players, and their expectations. This includes everything from the expectation of communication back to the referring doctor to medical record keeping. In addition, hierarchical models require that each hypnosis practitioner understand his/her expected role within that organizational structure and take the steps to optimize that role. For example, psychologists often can achieve at least affiliate status in most North American medical settings, placing them in a peer status with medical and nursing providers. For many complementary therapists, the challenge is more daunting. Forging personal relationships with medical providers, frequent communication about patient progress, and providing medical education for the clinic professionals can all serve to overcome prejudices and barriers.

2. Interprofessionalism paradigm. In those progressive settings where interprofessionalism is cultivated, it becomes important to cultivate effective communication skills and habits, for optimizing the communication across disciplinary and specialty lines. In addition, it is critical that hypnotic professionals NOT continue to think as solo practitioners when operating in an integrative setting. The patient's long-term wellbeing will be improved most effectively with a collaborative involvement of several disciplines in a coordinated care plan. Hypnosis may also be provided by multiple specialties, and the well trained and certified practitioner can multiply her or his impact by training nurses, EMTs, and others whose scope of practice allows the use of hypnosis.

3. Smorgasbord paradigm. In settings where the patients seem to be selecting complementary therapies from a smorgasbord, the challenge for hypnosis is patient education and marketing. Any brochures or videos presenting treatment options for patients should be reviewed, so that hypnosis is presented accurately but also attractively. Can the practitioner or a 
health educator convey a good grasp of the potential value of hypnosis in patient orientations, in a fashion that corrects misconceptions and awakens hope?

4. Lifestyle medicine paradigm. In accordance with the lifestyle medicine model, the challenge for hypnosis practitioners is to educate both peers and patients about the value of hypnosis for facilitating behavior change. Further, the lifestyle medicine paradigm suggests that hypnosis in the office can be supplemented by simultaneous patient-directed behavioral and lifestyle changes. For example, research shows that hypnosis can greatly moderate symptoms of irritable bowel syndrome [71], yet corresponding dietary changes can also facilitate long term digestive health [72]. The hypnosis practitioner can coordinate care with functional medicine physicians, dieticians, exercise physiologists, and complementary medicine therapists to develop a comprehensive treatment program. Lifestyle changes accompanying hypnotic treatment will also support patient wellbeing long after hypnosis sessions cease.

5. Health and wellness coaching paradigm. Health and wellness coaches specialize in facilitating active patient engagement in treatment, autonomous goal setting, and effective behavior change. Health and wellness coaches are welcomed by at least one major hypnosis organization in North America today (the Society for Clinical and Experimental Hypnosis) and can learn hypnotic techniques that can be used in service of lifestyle change to assist patient's selfdirected change. Their primary model remains collaborative health-enhancing goal setting [50], but hypnotic and self-hypnotic techniques can be introduced to assist the patient in overcoming obstacles and achieving goals.

6. Functional medicine paradigm. Understanding the functional medicine paradigm primarily challenges the hypnosis practitioner toward cross-disciplinary coordination of care. As mentioned, high stress levels can adversely food choices, digestion, and metabolism. Accordingly, dietary integrated with hypnotically-assisted stress reduction may more effectively treat many disorders. In McGrady and Moss [2], we included a case history of a female patient with lupus systemic erythematosus, who benefitted greatly from a coordinated program of integrative care including both hypnosis and functional medicine. The functional medicine interventions appeared at the time to have the greatest impact, to the extent that the patient's antinuclear antibodies normalized and her lupus symptoms were significantly reduced. The patient coped with occasional "flare" in her lupus symptoms by a combination of nutritional interventions, paced breathing, selfhypnosis, and self-soothing imagery.

7. Competencies paradigm. Competency-based education is applicable in hypnosis training and education. Appropriate competencies include mastery of clinical hypnosis intervention skills, and also the communication, cultural sensitivity, and related competencies described above.

8. Eco-systems paradigm. Hypnosis practitioners frequently focus on the inner world, utilizing hypnotic experiences to reverse the impact of external stress, resolve emotional conflicts, abreact emotion-laden memories, and enhance patient resilience and coping. Yet the question remains, whether the patient is returning to a toxic "morbidogenic" environment, toxic in the sense of physico-chemical hazards or emotionally destructive interactions. The eco-systems approach is a timely reminder to pay attention to the patient's inner and outer world, seek strategies to reduce exposure to harmful surroundings and increase patient time spent in salutogenic and supportive surroundings.

9. A word about research. The research on hypnotic interventions is encouraging but not conclusive. Hypnosis research is not supported by pharmaceutical profits; many hypnosis 
researchers conduct pilot studies lacking controls or controlled studies with small samples. Moving into the evidence-based world of integrative medicine, improvements in research design, collaborative studies among several sites to increase sample size, preregistration of protocols, and other steps to enhance the credibility of hypnosis research are priorities. A Task Force to Establish Efficacy Standards in Hypnosis Research is currently underway, supported by the boards of the International Society of Hypnosis, Division 30 of the American Psychological Association, the Society of Clinical and Experimental Hypnosis, the American Society of Clinical Hypnosis, the National Pediatric Hypnosis Training Institute, and the Milton Erickson Foundation. Researchers from six countries have been meeting since February 2019 to address efficacy research standards [73].

\section{Conclusion}

In summary, if hypnosis professionals desire a place at the ever-growing table of integrative healthcare, it is time to rethink hypnosis practice. Those who are already conditioned by years of solo practice may benefit by arranging to consult and spend time in the closest integrative setting. Fortunately, many early career professionals are already growing up professionally in integrative settings, and interprofessional collaboration and communication may be their native tongue.

\section{Author Contributions}

Donald Moss authored the document in entirety. He acknowledges help in locating relevant publications from his research assistant Shannon McLain Sims, currently a post-doctoral fellow at Saybrook University.

\section{Competing Interests}

The author has declared that no competing interests exist.

\section{References}

1. McGrady A, Moss D. Pathways to illness, pathways to health. New York, NY: Springer; 2013.

2. McGrady A, Moss D. Integrative pathways: Navigating chronic illness with a mind-body-spirit approach. New York, NY: Springer; 2018.

3. Moss D, McGrady A, Davies T, Wickramasekera I. Handbook of mind-body medicine in primary care. Thousand Oaks, CA: Sage Publications; 2003.

4. Weisfeld V. Summit on integrative medicine and the health of the public: Issue, background, and overview. Washington, DC: Institute of Medicine; 2009. Available from: http://www.bravewell.org/integrative_medicine/

5. Engel G. The need for a new medical model: A challenge for biomedicine. Science. 1977; 196: 129-136.

6. World Health Organization. Global action plan for the prevention and control of NCDs 20132020. Geneva, Switzerland: WHO; 2013.

7. World Health Organization. Non-communicable diseases: Fact sheet. Geneva, Switzerland: WHO; 2015. 
8. World Health Organization. Management of non-communicable diseases. Geneva, Switzerland: WHO; 2016.

9. Koenig HG. MSJAMA: Religion, spirituality, and medicine: Application to clinical practice. JAMA. 2000; 284: 1708.

10. Weil A. What is $I M / I H$ ? Andrew Weil Center for Integrative Medicine; 2020. Available from: https://integrativemedicine.arizona.edu/about/definition.html

11. World Health Organization. Constitution of the World Health Organization. Geneva, Switzerland: WHO; 1946.

12. De Benedettis G, Mammini C, Rago N. Blue book: La guida all'ipnosi evidence based. (Blue book: A guide to evidence-based hypnosis). Milan, Italy: Franco Angeli Publishers; 2018.

13. Elkins G. Clinician's guide to medical and psychological hypnosis: Foundations, applications, and professional issues. New York, NY: Springer; 2017.

14. Mendoza M, Capafons A. Eficacia de la hipnosis clínica: Resumen de su evidencia empírica. Papeles del psicólogo: revista del Colegio Oficial de Psicólogos. 2009; 30: 98-116.

15. Thompson T, Terhune DB, Oram C, Sharangparni J, Rouf R, Solmi M, et al. The effectiveness of hypnosis for pain relief: A systematic review and meta-analysis of 85 controlled experimental trials. Neurosci Biobehav R. 2019; 99: 298-310.

16. McEwen BS. Integrative medicine: Breaking down silos of knowledge and practice an epigenetic approach. Metabolism. 2017; 69: 21-29.

17. Carmody TP, Duncan C, Simon JA, Solkowitz S, Huggins J, Lee S, et al. Hypnosis for smoking cessation: A randomized trial. Nicotine Tob Res. 2008; 10: 811-818.

18. Carmody TP, Duncan CL, Solkowitz SN, Huggins J, Simon JA. Hypnosis for smoking relapse prevention: A randomized trial. Am J Clin Hyp. 2017; 60: 159-171.

19. Munson SO, Barabasz AF, Barabasz M. Ability of hypnosis to facilitate movement through stages of change for smoking cessation. Int J Clin Exp Hyp. 2018; 66: 56-82.

20. Green JP, Lynn SJ. A multifaceted hypnosis smoking-cessation program: Enhancing motivation and goal attainment. Int J Clin Exp Hyp. 2017; 65: 308-335.

21. Lang EV, Berbaum KS, Faintuch S, Hatsiopoulou O, Halsey N, Li X, et al. Adjunctive selfhypnotic relaxation for outpatient medical procedures: A prospective randomized trial with women undergoing large core breast biopsy. Pain. 2006; 126: 155-164.

22. Montgomery GH, Bovbjerg DH, Schnur JB, David D, Goldfarb A, Weltz CR, et al. A randomized clinical trial of a brief hypnosis intervention to control side effects in breast surgery patients. J Natl Cancer I. 2007; 99: 1304-1312.

23. Montgomery GH, David D, Kangas M, Green S, Sucala M, Bovbjerg DH, et al. Randomized controlled trial of a cognitive-behavioral therapy plus hypnosis intervention to control fatigue in patients undergoing radiotherapy for breast cancer. J Clin Oncol. 2014; 32: 557-563.

24. Jensen MP. Hypnosis for chronic pain management: Therapist guide. New York, NY, US: Oxford University Press; 2011.

25. Jensen MP. Hypnotic techniques for acute pain management: Favorite methods of master clinicians. Kirkland, WA: Denny Creek Press; 2019.

26. Ajam AA, Nguyen XV, Kelly RA, Ladapo JA, Lang EV. Effects of interpersonal skills training on MRI operations in a saturated market: A randomized trial. J Am Coll Radiol. 2017; 14: 963-970.

27. Lang E. Comfort talk: From the waiting room to the treatment suite. Dtsch Z Zahnarztl Hypn. 2019; 25: 22-24. 
28. Stuart JH. Hypnosis in contemporary medicine. Mayo Clin Proc. 2005; 80: 511-524.

29. National Institutes of Health. Complementary, alternative, or integrative health: What's in a name? 2019. Available from: https://nccih.nih.gov/health/integrative-health

30. Clarke TC, Black LI, Stussman BJ, Barnes PM, Nahin RL. Trends in the use of complementary health approaches among adults: United States, 2002-2012. National Health Statistics Reports. 2015; 79: 1-16.

31. Qaseem A, Wilt TJ, McLean RM, Forciea MA. Noninvasive treatments for acute, subacute, and chronic low back pain: A clinical practice guideline from the American College of Physicians. Ann Intern Med. 2017; 166: 514-530.

32. Lake J. Integrative mental health care: From theory to practice, part 1. Altern Ther Health M. 2007; 13: 50-56.

33. Lake J, Helgason C, Sarris J. Integrative mental health (IMH): Paradigm, research, and clinical practice. Explore. 2012; 8: 50-57.

34. Lake J. Integrative mental health care. New York, NY: W. W. Norton \& Company, Inc; 2009.

35. Rosenthal B, Lisi AJ. A qualitative analysis of definitions of integrative medicine and health. Top Integr Healthc. 2014; 5: 4.

36. Weeks J. What is the commitment to interprofessionalism in integrative health and medicine? Glob Adv Health Med. 2015; 4: 9-11.

37. World Health Organization. Framework for action on interprofessional education and collaborative practice. Geneva. Switzerland: WHO; 2010.

38. Scarton LA, Del Fiol G, Oakley-Girvan I, Gibson B, R. L, Workman TE. Understanding cancer survivors' information needs and information-seeking behaviors for complementary and alternative medicine from short- to long-term survival: A mixed-methods study. J Med Libr Assoc. 2018; 106: 87-97.

39. Balneaves LG, Weeks L, Seely D. Patient decision-making about complementary and alternative medicine in cancer management: Context and process. Curr Oncol. 2008; 15: 94100.

40. Jensen P. Transgenerational epigenetic effects on animal behaviour. Prog Bio Mol Bio. 2013; 113: 447-454.

41. Sagner M, Katz D, Egger G, Lianov L, Schulz KH, Braman M, et al. Lifestyle medicine potential for reversing a world of chronic disease epidemics: From cell to community. Int J Clin Pract. 2014; 68: 1289-1292.

42. Ornish D, Scherwitz LW, Billings JH, Brown SE, Gould KL, Merritt TA, et al. Intensive lifestyle changes for reversal of coronary heart disease. JAMA. 1998; 280: 2001-2007.

43. Silberman A, Banthia R, Estay IS, Kemp C, Studley J, Hareras D, et al. The effectiveness and efficacy of an intensive cardiac rehabilitation program in 24 sites. Am J Health Promot. 2010; 24: $260-266$.

44. Ornish D. Dr. Dean Ornish's program for reversing heart disease. New York, NY: Ivy Books/Ballantine Books; 1996.

45. Pischke CR, Frenda S, Ornish D, Weidner G. Lifestyle changes are related to reductions in depression in persons with elevated coronary risk factors. Psychol Health. 2010; 25: 10771100. 
46. Katz DL. Lifestyle as medicine: Culture, collusion, and pseudo-collusion. US News and World Report. 2015. Available from: https://news.yahoo.com/lifestyle-medicine-culture-collusionpseudo-confusion-174308408.html

47. Kini V, Ho PM. Interventions to improve medication adherence: A review. JAMA. 2018; 320: 2461-2473.

48. Jones DE, Carson KA, Bleich SN, Cooper LA. Patient trust in physicians and adoption of lifestyle behaviors to control high blood pressure. Patient Educ Couns. 2012; 89: 57-62.

49. Arloski M. Wellness coaching for lasting lifestyle change. Duluth, $M N$ : Whole Person Associates; 2007.

50. Jordan M. Integrative health coaching. Novato, CA: Global Medicine Hunter; 2018.

51. Ryan R, Deci E. An overview of self-determination theory: An organismic dialectical perspective. In: Handbook of self determination research. Rochester: The University of Rochester Press; 2002: 3-36.

52. Phelps C. The lived experience of individuals with Type 2 diabetes who have sustained successful lifestyle change and achieved long-term positive health outcomes: A detailed look at the female experience (Doctoral Dissertation). San Francisco: Saybrook University; 2014.

53. Prochaska JO, Norcross JC. Stages of change. Psychother. 2001; 38: 443-448.

54. Jones DS, Baker SM, Bennet P, Bland JS, Hedeya RJ, Galland J. Textbook of functional medicine. Gig Harbor, WA: Institute for Functional Medicine; 2010.

55. Haller D. The gut microbiome in health and disease. Cham, Switzerland: Springer; 2018.

56. Locke AE, Kahali B, Berndt SI, Justice AE, Pers TH, Day FR, et al. Genetic studies of body mass index yield new insights for obesity biology. Nature. 2015; 518: 197-206.

57. Zhang X, Qi Q, Zhang C, Smith SR, Hu FB, Sacks FM, et al. FTO genotype and 2-year change in body composition and fat distribution in response to weight-loss diets: The pounds lost trial. Diabetes. 2012; 61: 3005-3011.

58. Florez JC, Jablonski KA, Bayley N, Pollin TI, de Bakker PIW, Shuldiner AR, et al. TCF7L2 polymorphisms and progression to diabetes in the diabetes prevention program. New Engl J Med. 2006; 355: 241-250.

59. Keefer L, Blanchard EB. The effects of relaxation response meditation on the symptoms of irritable bowel syndrome: Results of a controlled treatment study. Behav Res Therapy. 2001; 39: 801-811.

60. Pirbaglou M, Katz J, de Souza RJ, Stearns JC, Motamed M, Ritvo P. Probiotic supplementation can positively affect anxiety and depressive symptoms: A systematic review of randomized controlled trials. Nutr Res. 2016; 36: 889-898.

61. Yau Y, Potenza M. Stress and eating behaviors. Minerva Endocrinol. 2013; 38: 255-267.

62. Kiecolt-Glaser JK. Stress, food, and inflammation: Psychoneuroimmunology and nutrition at the cutting edge. Psychosom Med. 2010; 72: 365-369.

63. Miller BF, Gilchrist EC, Kaile KM, Ross KM, Wong SL, Blount A, et al. Core competencies for behavioral health providers working in primary care. Prepared from the Colorado Consensus Conference. 2016.

64. Hoge MA, Morris JA, Laraia M, Pomerantz A, Farley T. Core competencies for integrated behavioral health and primary care. Washington, DC: SAMHSA - HRSA Center for Integrated Health Solutions; 2014. 
65. Pruss-Ustun A, Wolf J, Corvalan C, Neville T, Bos R, Neira M. Diseases due to unhealthy environments: An updated estimate of the global burden of disease attributable to environmental determinants of health. J Pub Health. 2017; 39: 464-475.

66. Emerson RW, Cameron KW. Nature (1836): Scholars' facsimiles \& reprints; 1940.

67. Miyazaki Y, Ikei H, Song C. Forest medicine research in Japan. JP J Hyg. 2014; 69: 122-135.

68. Li Q. Effect of forest bathing trips on human immune function. Environ Health Prev. 2010; 15 : 9-17.

69. Jia BB, Yang ZX, Mao GX, Lyu YD, Wen XL, Xu WH, et al. Health effect of forest bathing trip on elderly patients with chronic obstructive pulmonary disease. Biomed Environ Sci. 2016; 29: 212-218.

70. Hartl A. Woodland-an effective remedy? Myth vs. evidence-based medicine. Keynote presentation to the second international congress on forest and its potential for health. Krems, Austria; 2018.

71. Palsson OS. Hypnosis treatment of gastrointestinal disorders: A comprehensive review of the empirical evidence. Am J Clin Hyp. 2015; 58: 134-158.

72. Hill P, Muir JG, Gibson PR. Controversies and recent developments of the low-fodmap diet. Gastroenterol Hepatol. 2017; 13: 36-45.

73. Moss D. Task force to establish efficacy standards in hypnosis research. Focus. 2019; 61: 3.

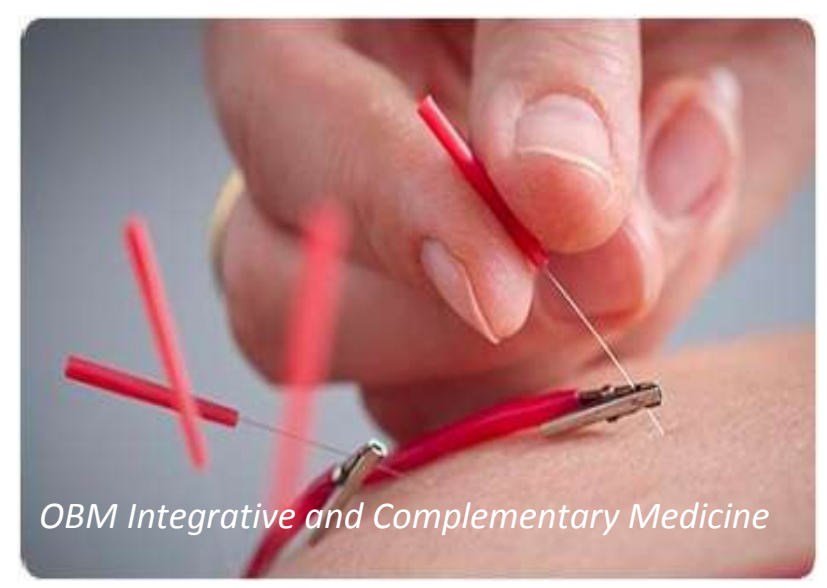

Enjoy OBM Integrative and Complementary Medicine by:

1. Submitting a manuscript

2. Joining in volunteer reviewer bank

3. Joining Editorial Board

4. Guest editing a special issue

For more details, please visit: http://www.lidsen.com/iournals/icm 